\title{
Saúde bucal e o desempenho diário de adultos em Chapecó, Santa Catarina, Brasil
}

\author{
Oral health and daily performance in adults in \\ Chapecó, Santa Catarina State, Brazil
}

\author{
1 Faculdade de Medicina, \\ Universidade de São Paulo, \\ São Paulo, Brasil. \\ 2 Centro de Ciências da \\ Saúde, Universidade \\ Federal de Santa Catarina \\ Florianópolis, Brasil. \\ Correspondência \\ J. T. Lacerda \\ Departamento de Saúde \\ Pública, Centro de Ciências \\ da Saúde, Universidade \\ Federal de Santa Catarina \\ Rua Vera Linhares de \\ Andrade 1968, casa 11, \\ Florianópolis, SC \\ 88034-700, Brasil. \\ jtelino@gmail.com
}

\section{Abstract}

The use of subjective indicators in dentistry has been increasing. This was a population-based cross-sectional study in the city of Chapecó, Santa Catarina State, Brazil, analyzing the relationship between oral health conditions and quality of life in 35-44-year-old adults ( $n=622)$. A further objective was to observe the influence of habits, dental visits, self-evaluation of health status, and socio-demographic characteristics in this process. The sample was selected using a multiplestage approach. Trained professionals collected the data. A multiple non-conditional, hierarchical logistic regression model was used to analyze the determinants of low (Oral Impact on Daily Performance - OIDP $\leq 3)$ and high $(O I D P \geq 10)$ interference of oral health in daily routine. The response rate was $81 \%$. Oral health status was found to interfere in the daily routine of $20.7 \%$ of participants and was more severe for $11.4 \%$. Household crowding, low-income neighborhoods, female gender, and orofacial pain in the previous six months were associated with higher interference in quality of life. The use of subjective indicators in public health services planning and definition of priority groups for delivery of oral health services is highly recommended.

Oral Health; Quality of Life; Adult; Health Inequalities
Josimari Telino de Lacerda 1,2

Euclides Ayres de Castilho 1

Maria Cristina Marino Calvo 2

Sérgio Fernando Torres de Freitas 2

\section{Introdução}

A histórica utilização, quase exclusiva, de indicadores clínicos para analisar a condição de saúde bucal de grupos populacionais e para o planejamento e avaliação de serviços de saúde é um aspecto em constante discussão no meio odontológico. A ênfase nesses indicadores, segundo Chen \& Hunter 1 , superdimensiona a visão normativa do processo saúde-doença, mascarando as suas dimensões sociais. Concomitantemente, discute-se o limitado valor das estratégias de mudança de hábitos e de comportamentos que orientam as práticas de promoção de saúde na melhoria das condições de saúde bucal 2,3.

Recentemente, autores como Holst \& Schüller 4 têm apontado para a influência do contexto social sobre os aspectos comportamentais e psicológicos, e destes últimos sobre o processo biológico em saúde bucal, com destaque para a cárie dentária. Um significativo número de investigações evidenciou a relação entre piores condições sócio-econômicas e precárias condições de saúde bucal, com percentuais de explicação variando entre $25 \%$ e $88 \%{ }^{5}$. O relato sobre saúde bucal apresentado pela Organização Mundial da Saúde (OMS), em 2003, evidenciou a influência dos fatores sócio-culturais e dos aspectos ambientais sobre a condição de saúde bucal, com piores resultados entre grupos com precária condição de vida e com tradições, crenças e cultura, as quais não favorecem a saúde oral 6 . 
Por outro lado, é crescente o interesse sobre o tema qualidade de vida em estudos de saúde geral 7,8 e de saúde bucal 1,5,9,10. Sheiham et al. ${ }^{9}$ consideram a qualidade de vida dos indivíduos fortemente influenciada por sua condição de saúde bucal. Restrições físicas e psicológicas podem influenciar diretamente em aspectos da alimentação, fala, locomoção, convívio social e auto-estima.

Locker 10, no entanto, adverte para o fato de piores indicadores clínicos não refletirem, necessariamente, relatos de impacto negativo na qualidade de vida. Segundo o autor, é possível encontrar indivíduos portadores de enfermidades crônicas relatando melhores níveis de qualidade de vida.

Allison et al. ${ }^{11}$ sugerem, como explicação desse fenômeno, a característica multidimensional e subjetiva do construto qualidade de vida. Outros autores concordam com a idéia de que a natureza freqüentemente assintomática das morbidades e agravos bucais, bem como o receio, a ansiedade ou mesmo a aversão ao tratamento odontológico podem ainda contribuir para explicar esse comportamento. Os autores argumentam que ansiedade e impacto decorrem de características psicológicas, envolvendo aspectos de afetividade negativa. Por outro lado, consideram que maior negligência dos indivíduos com sua condição bucal geraria maior volume e complexidade nos tratamentos, implicando o aumento da ansiedade, a diminuição da procura, a piora da condição bucal e, portanto, o maior impacto 12,13.

Leão \& Sheiham ${ }^{14}$ ressaltam que diferentes níveis de saúde bucal proporcionam diferentes reflexos no cotidiano das pessoas e que é desejável que, no estudo das necessidades de saúde bucal dos indivíduos, as dimensões sociais e psicossociais sejam consideradas simultaneamente com a condição clínica. Reisine et al. 15, ao analisarem a adequação do uso de indicadores de qualidade de vida na odontologia, concluíram que os mesmos são sensíveis na identificação e na descrição do impacto em diferentes condições clínicas bucais.

O presente estudo teve como objetivos analisar o grau em que a saúde bucal compromete o desempenho diário referido por adultos e identificar as variáveis de condição de vida, de percepção da condição de saúde, dos hábitos e dos cuidados e de condição de saúde bucal que melhor explicam interferências na qualidade de vida desses indivíduos, utilizando um modelo hierárquico de determinação. A hipótese que orientou a investigação é de que a condição de saúde bucal interfere no cotidiano das pessoas com reflexos sobre a qualidade de vida e é influenciada pelos hábitos e cuidados, pela percep- ção da condição de saúde e pelas características sócio-demográficas dos indivíduos.

\section{Método}

Realizou-se um estudo transversal de base populacional em adultos de 35 a 44 anos de idade, residentes na área urbana do Município de Chapecó, Santa Catarina, Brasil, no ano de 2004. Distante $569 \mathrm{~km}$ da capital do estado, Chapecó é o principal pólo econômico da região oeste catarinense, com economia baseada na agroindústria, com $91,6 \%$ da sua população residindo em área urbana 16.

O tamanho mínimo da amostra foi estabelecido visando estimar parâmetros com prevalências $\mathrm{p}=0,50$, valor de proporção com maior magnitude de variância, com um intervalo de 95\% de confiança (IC95\%) e um erro bilateral de $5 \%$. Considerando um efeito do desenho igual a 1,5 e possíveis não-respostas e perdas (10\%), o tamanho final da amostra foi de 622 indivíduos 17. O desenho da amostragem teve as seguintes etapas: a partir de dados do Censo Demográfico 2000 (Instituto Brasileiro de Geografia e Estatística, http://www.ibge.gov.br), pelo procedimento de análise de agrupamentos, técnica de K-means 18 , foram obtidos três aglomerados homogêneos - grupos; um total de 50 setores censitários foi selecionado proporcionalmente ao número de setores em cada grupo; em cada grupo, foram selecionados " $\mathrm{n}$ " indivíduos proporcionalmente ao número total de pessoas na faixa etária de 35-44 anos; seleção sistemática de domicílios em cada setor; e seleção, com igual probabilidade, de um indivíduo em cada domicílio. Os grupos podem ser assim caracterizados: Grupo 1: analfabetismo, escolaridade baixa e renda baixa; Grupo 2: escolaridade alta, renda alta e instalação sanitária adequada; e Grupo 3: domicílios próprios, abastecimento de água adequado e coleta de lixo.

A identificação dos domicílios e dos indivíduos baseou-se nos mapas de territorialização e no banco de dados do cadastro da Estratégia Saúde da Família.

Para garantir estimativas de intervalos com variâncias robustas, levando em consideração os possíveis efeitos de conglomerado na obtenção da amostra, recorreu-se ao comando logistic com as opções robust e cluster do programa Stata versão 9.0 (Stata Corp., College Station, Estados Unidos).

Os dados primários foram coletados por meio de entrevista e exames clínicos bucais com o auxílio de espelhos clínicos, no interior do domicílio, com os indivíduos sentados próximo a 
uma fonte de luz natural. As informações foram anotadas em ficha clínica própria. As entrevistas antecederam aos exames clínicos, sendo ambos realizados em uma única oportunidade para cada indivíduo. A coleta dos dados foi realizada nos períodos matutino e vespertino, em todos os dias da semana, durante três meses consecutivos.

Participaram da coleta de dados dez pessoas, compondo quatro equipes de trabalho, integradas por examinadores anotadores e monitores, treinados e calibrados segundo protocolo recomendado por Peres et al. 19. Adotou-se 0,61 como o valor mínimo de concordância ajustada aceita entre os participantes 20. Pré-teste dos instrumentos e estudo piloto foram realizados para se atingir as recomendações.

A variável dependente, grau de interferência da condição bucal no desempenho diário, determinada pelo índice Oral Impacts on Daily Performances (OIDP) 21, foi dicotomizada em baixa $(\mathrm{OIDP}<3)$ e alta interferência (OIDP $\geq 10,0)$. O valor médio do OIDP na amostra e a mediana do OIDP entre os indivíduos que referiram alguma interferência definiram os pontos de corte dos grupos de baixa e de alta interferência, respectivamente.

As variáveis idade (anos completos), sexo, estado marital (casado, solteiro, divorciado, viúvo), escolaridade (número de anos de estudo), somatório da renda mensal familiar em salários mínimos, situação de trabalho (trabalhador do setor formal, trabalhador do setor informal, trabalhadora do lar, sem trabalho, estudante/ aposentado/pensionista), tipo de moradia (alvenaria, madeira, mista, outro material), fonte de abastecimento de água no domicílio (abastecimento público, outra fonte) e aglomeração por domicílio (número de pessoas por cômodo do domicílio), constituíram as características sócio-demográficas. Avaliação da saúde (excelente, muito boa, boa, razoável e ruim), dor orofacial nos últimos seis meses 22, hábitos e recursos de higiene bucal (freqüência de escovação dental diária, uso de creme dental, uso de fio dental), freqüência do consumo de doces entre as refeições, acesso a serviços odontológicos nos últimos 12 meses, local (público, particular/outros) e motivo da consulta no último ano (dor, revisão, limpeza, exodontia, restauração, outros) definiram o eixo percepção da condição de saúde, dos hábitos e dos cuidados referentes à saúde bucal e acesso a serviços odontológicos. O eixo condição bucal foi definido pelas variáveis: condição coronária para cárie dentária (CPO-D) e necessidade de tratamento, condição periodontal pelo índice periodontal comunitário (CPI) e pelo índice de perda de inserção (IPI), perdas dentárias (segun- do número de dentes perdidos e localização por arco dental e anterior ou posterior, uso e necessidade de próteses dentárias), todas segundo os critérios recomendados pela OMS em 199723. Optou-se por coletar separadamente os agravos periodontais permitindo a análise, em particular, do sangramento gengival (presente, ausente), bolsa periodontal rasa (presente, ausente), bolsa periodontal profunda (presente, ausente), perda de inserção (presente, ausente).

Para análise e inclusão no modelo, algumas variáveis foram categorizadas: estado marital (casado, não casado), escolaridade (8 anos ou mais, menos de 8 anos), renda familiar (acima de 3 salários mínimos, até 3 salários mínimos), tipo de moradia (alvenaria, outros), aglomeração no domicílio (mais de 1 pessoa/cômodo, até 1 pessoa/cômodo), avaliação da saúde (excelente/ muito boa/boa, razoável/ruim), dor orofacial no último semestre (presente, ausente), freqüência de escovação dental (3 ou mais vezes/dia, menos de 3 vezes/dia), consumo de doce entre as refeições (sim, não), necessidade de tratamento por cárie (nenhum, algum), componente cariado do CPO-D (nenhum e maior ou igual a 3 dentes), componente perdido do CPO-D (maior que quatro dentes, menor ou igual a quatro dentes), uso de prótese (sim, não) e necessidade de prótese (sim, não).

A estatística -teste de qui-quadrado foi usada na análise da associação seguida da estimativa da odds ratio (OR) e respectivos IC95\%. Na análise dos fatores de determinação para a gravidade da interferência, foram utilizados modelos de regressão logística múltipla, não condicional hierarquizada, seguindo os procedimentos de Hosmer \& Lemeshow 24.

O padrão hierárquico de determinação do processo saúde-doença proposto por Dahlgrem \&Whitehead 25 , adaptado para a variável dependente deste estudo, orientou a estruturação dos blocos de variáveis e conseqüentemente a ordem de entrada dos mesmos na modelagem. No modelo proposto, o bloco das variáveis sócioeconômicas constituiu os determinantes distais. Estas variáveis condicionaram os demais blocos de fatores determinantes: saúde auto-referida, hábitos e cuidados referentes à saúde bucal, acesso a serviços odontológicos (determinantes intermediários) e condição de saúde bucal (determinantes proximais) conforme a Figura 1. A observação dos fatores determinantes foi inicialmente realizada em cada bloco, em que as variáveis que apresentaram nível de significância descritivo $\mathrm{p}<0,20$ na análise bivariada foram inseridas no modelo logístico. Adotou-se o procedimento passo a passo para a seleção das variáveis (stepwise forward procedure). Op- 


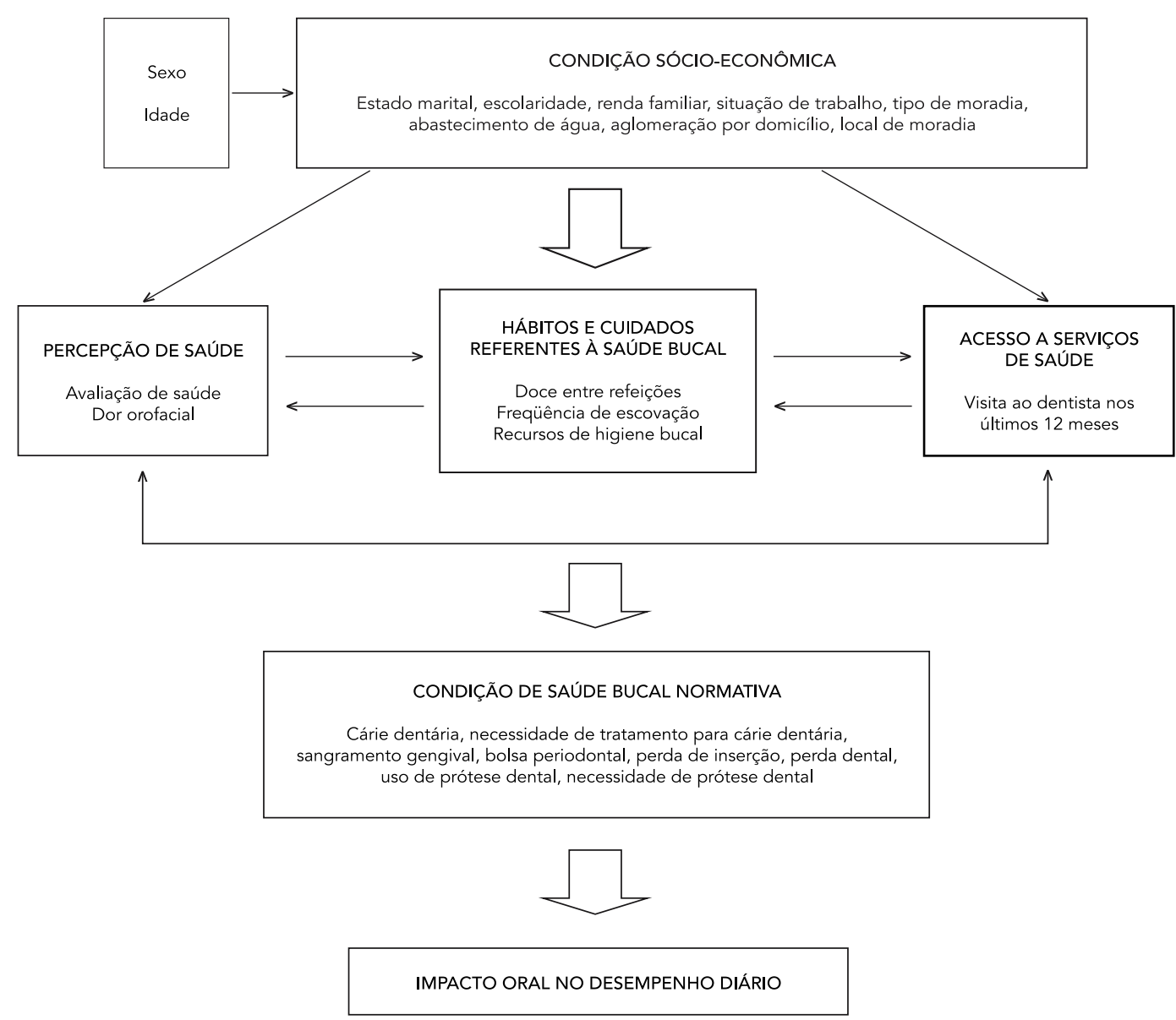

tou-se por manter as variáveis que apresentaram nível de significância $\mathrm{p}<0,05$ e/ou aceitas no ajuste do modelo. As variáveis sexo, renda familiar e escolaridade foram utilizadas como co-variáveis, independentemente do nível de significância estatística observado.

As variáveis mantidas em cada bloco de determinação compuseram a análise do modelo hierárquico. As variáveis do bloco da condição sócio-econômica foram as primeiras a serem incluídas no modelo, permanecendo como fatores de ajuste para os determinantes intermediários e proximais. O critério de permanência no modelo final foi o mesmo descrito para os modelos múltiplos de cada bloco, ou seja, com nível de significância $\mathrm{p}<0,05$ e/ou ajuste. Para avaliar o ajuste do modelo final, utilizou-se o teste de Hosmer \& Lemeshow. É oportuno salientar que, para esta análise, não se cogitou estabelecer o tamanho mínimo de amostra, constituindo-se, assim, uma limitação do estudo.

Participaram da pesquisa somente os indivíduos que firmaram o Termo de Consentimento Livre e Esclarecido. Foram assegurados o sigilo e o uso exclusivo das informações no estudo. Todos os procedimentos de biossegurança referentes ao indivíduo e aos examinadores foram obedecidos. O estudo foi aprovado pela Comissão de Ética para Análise de Projetos de Pesquisa do Hospital das Clínicas da Faculdade de Medicina da Universidade de São Paulo (registro no. 869/02). 


\section{Resultados}

A freqüência de resposta obtida foi de $81 \%$, totalizando 504 participantes. Os principais motivos das perdas foram recusa em participar da pesquisa $(13,6 \%)$ e não localização dos indivíduos, após três visitas em dias e horários diferentes $(5,4 \%)$.

Cinqüenta e nove por cento (IC95\%: 54,263,2 ) eram do sexo feminino, 52\% (IC95\%: 47,4$56,5)$ tinham entre 40 e 44 anos de idade, $81,1 \%$ (IC95\%: 77,4-84,5) eram casados, a metade (IC95\%: 46,8-56,9) informou ter menos de oito anos de estudos, e a renda média familiar referida foi de R\$ 817,40/mês (IC95\%: 759,40-875,30). O setor formal agregou a maioria dos entrevistados, correspondendo a 69,1\% (IC95\%: 63,8-74,2) dos indivíduos empregados. Em média, as residências apresentavam 3,9 cômodos (IC95\%: 3,8-4,0) e abrigavam quatro pessoas (IC95\%: 3,9-4,1). O índice de aglomeração foi menor ou igual a $1 \mathrm{em}$ 66,4\% (IC95\%: 62,0-70,7) dos casos entrevistados. Menos de 10\% (IC95\%: 7,0-12,5) dos domicílios eram desprovidos do abastecimento de água da rede pública.

Mais de dois terços (IC95\%: 65,7-74,0) avaliaram sua saúde como excelente ou boa, e 16,5\% (IC95\%: 13,3-20,2) informaram problemas de saúde no último ano. A prevalência de dor orofacial nos últimos seis meses foi 21,3\% (IC95\%: 17,7-25,2), dor de dente espontânea ou provocada, o tipo de dor mais freqüente (15,9\%; IC95\%: 12,8-19,5), e a intensidade leve ou moderada predominou em todos os tipos de dor investigados.

Quase toda a amostra escovava os dentes diariamente (95,2\%; IC95\%: 92,9-96,9), majoritariamente na freqüência de três vezes ou mais (89,6\%; IC95\%: 86,6-92,2), e fazia uso do creme dental (95,2\%; IC95\%: 92,9-96,9). Na limpeza dos espaços interdentais, o palito era utilizado por 79,5\% (IC95\%: 75,6-83,0) dos indivíduos, e o fio dental, por 49\% (IC95\%: 44,6-53,6).

Mais da metade (57,1\%; IC95\%: 52,6-61,6) visitou o dentista no último ano. O serviço público foi utilizado por 41,7\% (IC95\%: 35,8-47,7) dos que procuraram o dentista no último ano, seguido pelo setor privado (38\%; IC95\%: 32,3-44,0) e pelos serviços prestados pelas empresas, sindicatos e outros (20,3\%; IC95\%: 15,7-25,5).

\section{Condição de saúde bucal}

Quase todos os indivíduos apresentavam doença ou seqüelas do ataque de cárie dentária (98,7\%; IC95\%: 97,3-99,5). A média de dentes atacados foi igual a 18,1 (IC95\%: 17,6-18,6), sendo os componentes perdido $(\mathrm{P})$ e restaurado $(\mathrm{O})$ responsáveis por $95,3 \%$ da composição do CPO-D.
Cálculo dentário (90,3\%; IC95\%: 87,3-92,7) e sangramento gengival (57,3\%; IC95\%: 52,8-61,8) foram os agravos mais prevalentes na análise da condição periodontal. A periodontite estava presente em pouco menos de um quinto da população observada (IC95\%: 14,8-21,7), e menos de 4\% (IC95\%: 2,2-5,8) apresentaram periodontite grave. A perda de inserção acometia 30,6\% (IC95\%: 26,5-35,0) da população.

A maior necessidade de prótese dental se concentrava no arco inferior, afetando 69,3\% (IC95\%: 65,0-73,4) dos adultos examinados, enquanto, no arco superior, essa carência foi diagnosticada em 32,7\% (IC95\%: 28,5-37,1) das pessoas. O tipo de prótese mais indicado em ambos os arcos foi a prótese fixa ou prótese parcial removível, para reposição de mais de um elemento dentário.

\section{Interferência da condição bucal no desempenho diário}

A proporção de indivíduos que relataram alguma interferência da condição de saúde bucal nos últimos seis meses foi de 20,7\% (IC95\%: 17,2-24,6). O escore médio do OIDP foi 2,97 (desvio-padrão - $\mathrm{DP}=8,83$ ), e a média de atividades afetadas, igual a $0,51(\mathrm{DP}=1,27)$. Dentre as pessoas atingidas, o OIDP médio foi 14,17 (DP = 14,6), e a média de atividades afetadas igual a 2,43 (DP = 1,66). As atividades mais referidas foram comer e mastigar bem os alimentos (16,8\%; IC95\%: 13,5-20,46) e limpar os dentes e a boca (13,2\%; IC95\%: 10,316,6). Alta gravidade de interferência da saúde bucal no cotidiano, desfecho do presente estudo e arbitrariamente indicada como um valor de OI$\mathrm{DP} \geq 10,0$, foi referida por $11,4 \%$ (IC95\%: 8,7-14,6) dos participantes.

$\mathrm{Na}$ análise do bloco condição sócio-econômica e características demográficas, após o ajuste do modelo final, as variáveis aglomeração no domicílio, local de moradia e sexo permaneceram associadas à alta gravidade da interferência no desempenho de atividades cotidianas e foram selecionadas para a análise do modelo hierárquico. Residentes dos setores do Grupo $1(\mathrm{OR}=1,91$; IC95\%: 1,05-3,47), indivíduos que moravam em domicílios com mais de uma pessoa por cômodo $(\mathrm{OR}=2,12$; IC95\%: 1,15-3,90) e mulheres $(\mathrm{OR}=$ 2,10; IC95\%: 1,15-4,03) referiram maior gravidade que os demais (Tabela 1).

$\mathrm{Na}$ análise do bloco de determinação intermediária, as variáveis dor orofacial nos últimos seis meses, auto-avaliação de saúde e uso de fio dental apresentaram associação estatisticamente significante com alta gravidade de interferência nas atividades diárias, mantiveram-se associadas após o ajuste das variáveis dentro do bloco e foram incluídas no modelo final. A interferência 
Associação entre características sócio-demográficas e alta gravidade do índice Oral Impacts on Daily Performances (OIDP): modelo de regressão logística múltipla não condicional. Chapecó, Santa Catarina, Brasil, 2004

\begin{tabular}{|c|c|c|c|c|c|c|c|c|c|c|}
\hline \multirow[t]{2}{*}{ Variáveis } & \multicolumn{2}{|c|}{ Total } & \multicolumn{2}{|c|}{ OIDP $\geq 10,0$} & \multicolumn{2}{|c|}{ OIDP $<3,0$} & \multirow{2}{*}{$\begin{array}{c}\text { OR ** } \\
\text { (IC95\%) }\end{array}$} & \multirow{2}{*}{$\begin{array}{l}\text { Valor } \\
\text { de } p\end{array}$} & \multirow{2}{*}{$\begin{array}{l}\text { OR *** } \\
\text { (IC95\%) }\end{array}$} & \multirow{2}{*}{$\begin{array}{l}\text { Valor } \\
\text { de } p\end{array}$} \\
\hline & $\mathbf{N}$ & $\%$ * & $\mathbf{n}$ & $\%$ * & $\mathbf{n}$ & $\%$ * & & & & \\
\hline Sexo & & & & & & & & 0,004 & & 0,026 \\
\hline Masculino & 207 & 41,1 & 15 & 7,4 & 180 & 92,6 & 1,00 & & 1,00 & \\
\hline Feminino & 297 & 58,9 & 43 & 15,3 & 229 & 84,7 & $2,22(1,17-4,19)$ & & $2,10(1,15-4,03)$ & \\
\hline Escolaridade (anos) \# & & & & & & & & 0,01 & & 0,264 \\
\hline$\geq 8$ & 260 & 51,4 & 19 & 8,1 & 212 & 91,9 & 1,00 & & 1,00 & \\
\hline$<8$ & 244 & 48,6 & 39 & 16,2 & 197 & 83,8 & $2,18(1,20-3,97)$ & & $1,45(0,76-2,78)$ & \\
\hline \multicolumn{11}{|l|}{ Renda familiar } \\
\hline (salários mínimos) \#,\#\# & & & & & & & & 0,011 & & 0,257 \\
\hline Acima de 3 & 296 & 59,0 & 15 & 7,5 & 179 & 92,5 & 1,00 & & 1,00 & \\
\hline Até 3 & 208 & 41,0 & 43 & 15,8 & 226 & 84,2 & $2,30(1,21-4,35)$ & & $1,49(0,75-2,95)$ & \\
\hline Trabalho & & & & & & & & 0,241 & $\# \#$ & \#\#\# \\
\hline Setor formal & 222 & 46,2 & 21 & 9,7 & 190 & 90,3 & 1,00 & & & \\
\hline Setor informal & 99 & 17,4 & 12 & 13,8 & 72 & 86,2 & $1,50(0,69-3,29)$ & & & \\
\hline Do lar & 92 & 18,4 & 10 & 11,8 & 77 & 88,2 & $1,21(0,54-2,73)$ & & & \\
\hline Sem trabalho & 60 & 11,8 & 12 & 21,2 & 43 & 78,8 & $2,54(1,13-5,68)$ & 0,024 & & \\
\hline Outros & 31 & 6,1 & 3 & 10,7 & 27 & 89,3 & $1,00(0,28-3,84)$ & & & \\
\hline Tipo de moradia & & & & & & & & 0,129 & \#\#\# & \#\#\# \\
\hline Alvenaria & 256 & 50,9 & 24 & 10,0 & 216 & 90,0 & 1,00 & & & \\
\hline Outros & 248 & 49,1 & 34 & 14,5 & 193 & 85,5 & $1,56(0,89-2,77)$ & & & \\
\hline Água da rede & & & & & & & & 0,272 & $\# \#$ & \#\#\# \\
\hline $\operatorname{Sim}$ & 455 & 90,5 & 50 & 11,7 & 374 & 88,3 & 1,00 & & & \\
\hline Não & 49 & 9,5 & 8 & 17,1 & 35 & 82,9 & $1,61(0,69-3,80)$ & & & \\
\hline \multicolumn{11}{|l|}{ Aglomeração } \\
\hline (pessoa/cômodo) & & & & & & & & 0,001 & & 0,015 \\
\hline$\leq 1$ & 334 & 66,5 & 27 & 8,5 & 290 & 91,5 & 1,00 & & 1,00 & \\
\hline$>1$ & 170 & 33,5 & 31 & 19,7 & 119 & 80,3 & $2,69(1,56-4,78)$ & & $2,12(1,15-3,90)$ & \\
\hline Local de moradia & & & & & & & & 0,005 & & 0,033 \\
\hline Setores dos Grupos 2 e 3 & 328 & 67,2 & 28 & 9,2 & 280 & 90,8 & 1,00 & & 1,00 & \\
\hline Setores do Grupo 1 & 176 & 32,8 & 30 & 18,5 & 129 & 81,5 & $2,27(1,28-4,02)$ & & $1,91(1,05-3,47)$ & \\
\hline
\end{tabular}

Nota: teste de Hosmer \& Lemeshow $(p=0,780)$.

* Freqüência ponderada;

** Análise univariada;

*** Ajustada para as características demográficas e condição sócio-econômica entre si;

\# Mantida no modelo independentemente do nível de significância;

\#\# Salário mínimo em fevereiro de 2005 (R\$ 260,00);

\#\#\# Retirada do modelo por perda de significância estatística.

foi mais grave entre os indivíduos que apresentaram dor orofacial nos últimos seis meses $(\mathrm{OR}=$ 56,5; IC95\%: 24,3-131,5), avaliaram sua saúde, no último mês, como razoável ou ruim $(\mathrm{OR}=2,33$; IC95\%: 1,05-5,16) e não usavam fio dental diariamente (OR = 2,77; IC95\%: 1,22-6,35) (Tabela 2).

$\mathrm{Na}$ análise do bloco proximal, apresentaram associação independente com alta gravidade da interferência as seguintes condições bucais: perda de inserção do periodonto $(\mathrm{OR}=3,22$; IC95\%:
1,55-6,69) e presença de cárie dentária em 3 ou mais dentes (OR = 3,55; IC95\%: 1,55-8,15). Dados esses resultados, estas variáveis foram as representantes do bloco no modelo hierárquico. A variável necessidade de prótese dental foi considerada variável de confusão e mantida no modelo (Tabela 3).

A análise dos fatores determinantes para a alta gravidade da interferência no desempenho diário, no modelo hierárquico, evidenciou que 
Associação entre a alta gravidade do índice Oral Impacts on Daily Performances (OIDP) e a percepção da condição de saúde, hábitos e cuidados com saúde bucal e acesso a serviços de saúde: modelo de regressão logística múltipla não condicional. Chapecó, Santa Catarina, Brasil, 2004.

\begin{tabular}{|c|c|c|c|c|c|c|c|c|c|c|}
\hline \multirow[t]{2}{*}{ Variáveis } & \multicolumn{2}{|c|}{ Total } & \multicolumn{2}{|c|}{ OIDP $\geq 10,0$} & \multicolumn{2}{|c|}{ OIDP $<3,0$} & \multirow{2}{*}{$\begin{array}{c}\text { OR ** } \\
\text { (IC95\%) }\end{array}$} & \multirow{2}{*}{$\begin{array}{l}\text { Valor } \\
\text { de } p\end{array}$} & \multirow{2}{*}{$\begin{array}{c}\text { OR *** } \\
(\text { IC95\%) }\end{array}$} & \multirow{2}{*}{$\begin{array}{l}\text { Valor } \\
\text { de p }\end{array}$} \\
\hline & $\mathbf{N}$ & $\%$ * & $\mathbf{n}$ & $\%$ * & $\mathbf{n}$ & $\%$ * & & & & \\
\hline Doces entre as refeições & & & & & & & & 0,105 & $\#$ & \# \\
\hline Não & 423 & 84,0 & 44 & 11,1 & 347 & 88,9 & 1,00 & & & \\
\hline Sim & 81 & 16,0 & 14 & 17,6 & 62 & 82,4 & $1,75(0,89-3,43)$ & & & \\
\hline Fio dental & & & & & & & & 0,001 & & 0,015 \\
\hline Usa & 247 & 49,0 & 17 & 7,0 & 217 & 93,0 & 1,00 & & 1,00 & \\
\hline Não usa & 257 & 51,0 & 40 & 18,6 & 172 & 81,4 & $2,98(1,61-5,53)$ & & $2,77(1,22-6,35)$ & \\
\hline Avaliação da saúde & & & & & & & & $<0,001$ & & 0,038 \\
\hline Excelente/Boa & 354 & 70,1 & 25 & 7,3 & 305 & 92,7 & 1,00 & & 1,00 & \\
\hline Razoável/Ruim & 150 & 29,9 & 33 & 23,3 & 104 & 76,7 & $3,83(2,14-6,84)$ & & $2,33(1,05-5,16)$ & \\
\hline Dor orofacial & & & & & & & & $<0,001$ & & $<0,001$ \\
\hline Não & 396 & 78,7 & 9 & 2,2 & 378 & 97,8 & 1,00 & & 1,00 & \\
\hline Sim & 108 & 21,3 & 49 & 58,2 & 31 & 41,8 & $60,6(26,8-136,8)$ & & $56,5(24,3-131,5)$ & \\
\hline Visita ao dentista (último ano) & & & & & & & & 0,09 & $\#$ & $\#$ \\
\hline Não visitou & 216 & 42,8 & 18 & 8,8 & 184 & 91,2 & 1,00 & & & \\
\hline Particular/Outros & 121 & 23,1 & 15 & 11,5 & 139 & 88,5 & $1,40(0,64-3,03)$ & & & \\
\hline Público & 167 & 34,1 & 25 & 16,4 & 86 & 83,6 & $2,07(1,07-4,00)$ & & & \\
\hline
\end{tabular}

Nota: teste de Hosmer \& Lemeshow $(p=0,909)$.

* Freqüência ponderada;

** Análise univariada;

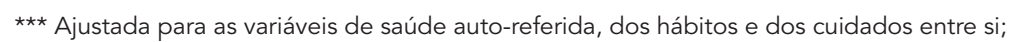

\# Retirada do modelo devido ao alto valor do nível de significância descrita.

mulheres (OR = 2,10; IC95\%: 1,09-4,03), residentes nas áreas do Grupo 1 (OR = 1,91; IC95\%: 1,05$3,47)$ e moradores em domicílios com mais de 1 pessoa por cômodo (OR = 2,12; IC95\%: 1,15-3,90) referiram maior gravidade que homens residentes nas áreas dos Grupos 2 e 3 e moradores em domicílios com menor número de pessoas por cômodo. No bloco intermediário, a auto-avaliação de saúde e a dor orofacial nos últimos seis meses foram fatores determinantes de maior gravidade, independente dos aspectos demográficos e da condição sócio-econômica. Pessoas que avaliaram sua saúde, nos últimos 12 meses, como regular ou ruim apresentaram quase o triplo de chance de referir a alta gravidade sobre os que avaliaram sua saúde como excelente ou boa (OR = 2,69; IC95\%: 1,18-6,15). O relato de alta gravidade da interferência da condição bucal no cotidiano foi maior entre os indivíduos que sentiram dor orofacial nos últimos seis meses que entre os que não sentiram no mesmo período (OR = 57,0; IC95\%: 23,69-137,34). Nenhuma condição de saúde bucal, segundo critérios normativos, manteve-se associada à alta gravidade da interferência após o ajuste pelos blocos anteriores. A variável necessidade de prótese foi mantida, para melhor ajuste do modelo final e por ser representativa de falta de dentes e problemas com a adaptação da prótese, elencada como segunda causa específica da interferência na perspectiva dos entrevistados (Tabela 4).

\section{Discussão}

O delineamento da amostra, o treinamento e a calibração dos pesquisadores, os exames em duplicatas e o controle de variáveis de confusão na análise dos dados foram estratégias utilizadas para assegurar a validade do estudo, a confiabilidade e reprodutibilidade dos dados.

O percentual de perdas de $19 \%$, apesar das diversas tentativas de busca adotadas, foi maior do que os $10 \%$ esperados, principalmente nos Grupos 2 e 3. Dados não apresentados mostraram inexistir diferenças significativas entre os indivíduos do universo amostral e os da população do estudo de maneira geral e dentro dos grupos, segundo sexo, renda e escolaridade. A propósito, esses achados reforçam a opção por tratar a 
Associação entre condição de saúde bucal segundo critérios normativos e alta gravidade do índice Oral Impacts on Daily Performances (OIDP): modelo de regressão logística múltipla não condicional. Chapecó, Santa Catarina, Brasil, 2004

\begin{tabular}{|c|c|c|c|c|c|c|c|c|c|c|}
\hline \multirow[t]{2}{*}{ Variáveis } & \multicolumn{2}{|c|}{ Total } & \multicolumn{2}{|c|}{ OIDP $\geq 10,0$} & \multicolumn{2}{|c|}{ OIDP $<3,0$} & \multirow{2}{*}{$\begin{array}{c}\text { OR ** } \\
\text { (IC95\%) }\end{array}$} & \multirow{2}{*}{$\begin{array}{l}\text { Valor } \\
\text { de } p\end{array}$} & \multirow{2}{*}{$\begin{array}{l}\text { OR *** } \\
\text { (IC95\%) }\end{array}$} & \multirow{2}{*}{$\begin{array}{l}\text { Valor } \\
\text { de } p\end{array}$} \\
\hline & $\mathbf{N}$ & $\%$ * & $\mathbf{n}$ & $\%$ * & $\mathbf{n}$ & $\%$ * & & & & \\
\hline Dente cariado & & & & & & & & $<0,001$ & & 0,003 \\
\hline Nenhum & 308 & 61,0 & 26 & 8,7 & 259 & 91,3 & 1,00 & & 1,00 & \\
\hline$\geq 3$ & 47 & 9,4 & 13 & 29,3 & 30 & 70,7 & $4,36(1,99-9,58)$ & & $3,55(1,55-8,15)$ & \\
\hline Dente perdido & & & & & & & & 0,18 & $\#$ & $\#$ \\
\hline$\leq 4$ & 149 & 27,3 & 12 & 8,8 & 137 & 91,2 & 1,00 & & & \\
\hline$>4$ & 318 & 72,7 & 46 & 14,0 & 272 & 86,0 & $1,63(0,79-3,32)$ & & & \\
\hline \multicolumn{11}{|l|}{ Necessidade de } \\
\hline tratamento por cárie & & & & & & & & 0,01 & $\#$ & $\#$ \\
\hline Nenhum & 314 & 62,3 & 27 & 8,9 & 263 & 91,1 & 1,00 & & & \\
\hline Algum & 190 & 37,7 & 31 & 17,2 & 146 & 82,8 & $2,10(1,19-3,72)$ & & & \\
\hline Sangramento gengival & & & & & & & & 0,074 & $\#$ & $\#$ \\
\hline Presente & 214 & 42,5 & 18 & 8,8 & 182 & 91,2 & 1,00 & & & \\
\hline Ausente & 290 & 57,5 & 40 & 14,7 & 227 & 85,3 & $1,73(0,95-3,17)$ & & & \\
\hline Bolsa periodontal profunda & & & & & & & & 0,011 & $\#$ & $\#$ \\
\hline Ausente & 485 & 96,2 & 52 & 11,3 & 398 & 88,7 & 1,00 & & & \\
\hline Presente & 19 & 3,8 & 6 & 33,3 & 11 & 66,7 & $3,87(1,36-11,00)$ & & & \\
\hline Perda de inserção & & & & & & & & 0,001 & & 0,002 \\
\hline Ausente & 349 & 69,3 & 28 & 8,7 & 292 & 91,3 & 1,00 & & 1,00 & \\
\hline Presente & 155 & 30,7 & 30 & 19,7 & 117 & 80,3 & $2,60(1,46-4,61)$ & & $3,22(1,55-6,69)$ & \\
\hline Necessidade de prótese & & & & & & & & 0,026 & & $0,122 \# \#$ \\
\hline Não & 128 & 25,2 & 8 & 5,9 & 117 & 94,1 & 1,00 & & 1,00 & \\
\hline Sim & 376 & 74,8 & 50 & 14,2 & 292 & 85,8 & $2,48(1,11-5,54)$ & & $2,26(0,80-6,39)$ & \\
\hline
\end{tabular}

Nota: teste de Hosmer \& Lemeshow $(p=0,628)$

* Freqüência ponderada;

** Análise univariada;

*** Ajustada para os aspectos clínicos de saúde bucal entre si;

\# Retirada do modelo por perda de significância estatística;

\#\# Mantida no ajuste do modelo.

amostra como sendo aleatória simples. Embora o tamanho final tenha sido menor que o previsto, esse aspecto correspondeu a um erro amostral estimado de $5,34 \%$ contra os $5 \%$ iniciais, o que não compromete os resultados.

Por outro lado, é necessário ter cautela ao se generalizar os resultados. A variável desfecho e algumas variáveis independentes relacionamse diretamente com a percepção, característica própria dos indivíduos investigados e dos aspectos culturais de cada grupo populacional, bem como envolve a memória do entrevistado. A permanência de um expressivo número de co-variáveis, decorrente dos critérios metodológicos adotados, constitui uma limitação deste estudo frente à possibilidade de existir colinearidade entre algumas variáveis. Outra limitação importante refere-se ao tipo de estudo. Todas as observações foram feitas em cada indivíduo, em uma única oportunidade, não sendo possível estabelecer a precedência temporal das variáveis independentes sobre o desfecho. Além disso, uma das grandes contribuições da odontologia para a sociedade é garantir a qualidade de vida por meio da prevenção e tratamento dos agravos bucais. Tais agravos afetam diretamente a capacidade mastigatória, a fala e, principalmente, acarretam sofrimento devido à sintomatologia dolorosa freqüentemente associada. Em adição, podem afetar a auto-estima e o bem-estar geral dos indivíduos ${ }^{5,9}$. Há consenso na literatura quanto a uma forte correlação entre a condição de saúde bucal e a qualidade de vida dos indivíduos 1,9,10,14. Condição bucal e capacidade de percepção influenciam diretamente essa relação. Considera-se que uma pior condição de saúde 
Resultados da análise de regressão logística múltipla não condicional hierarquizada para alta gravidade do índice Oral Impacts on Daily Performances (OIDP). Chapecó, Santa Catarina, Brasil, 2004.

\begin{tabular}{|c|c|c|c|c|}
\hline Modelo & OR * (IC95\%) & Valor de $\mathrm{p}$ & OR ** (IC95\%) & Valor de $\mathrm{p}$ \\
\hline \multirow{2}{*}{\multicolumn{5}{|c|}{$\begin{array}{l}\text { Características demográficas e } \\
\text { condição sócio-econômica }\end{array}$}} \\
\hline & & & & \\
\hline Sexo & & 0,004 & & 0,026 \\
\hline Masculino & 1,00 & & 1,00 & \\
\hline Feminino & $2,22(1,15-4,19)$ & & $2,10(1,09-4,03)$ & \\
\hline Escolaridade (anos de estudo) & & 0,01 & & $0,264 * \star \star$ \\
\hline$\geq 8$ & 1,00 & & 1,00 & \\
\hline$<8$ & $2,18(1,20-3,97)$ & & $1,456(0,76-2,78)$ & \\
\hline Renda familiar (salários mínimos) \# & & 0,011 & & $0,257 * \star \star$ \\
\hline Acima de 3 & 1,00 & & 1,00 & \\
\hline Até 3 & $2,30(1,21-4,22)$ & & $1,49(0,75-2,95)$ & \\
\hline Aglomeração (pessoa/cômodo) & & 0,005 & & 0,015 \\
\hline$\leq 1$ & 1,00 & & 1,00 & \\
\hline$>1$ & $2,69(1,56-4,78)$ & & $2,12(1,15-3,90)$ & \\
\hline Local de moradia & & 0,005 & & 0,033 \\
\hline Setores dos Grupos 2 e 3 & 1,00 & & 1,00 & \\
\hline Setores do Grupo 1 & $2,27(1,28-4,02)$ & & $1,91(1,05-3,47)$ & \\
\hline \multicolumn{5}{|l|}{ Percepção da condição de saúde, dos } \\
\hline \multicolumn{5}{|c|}{ hábitos e dos cuidados, acesso a serviços } \\
\hline Dor orofacial & & $<0,001$ & & $<0,001$ \\
\hline Não & 1,00 & & 1,00 & \\
\hline $\operatorname{Sim}$ & $60,6(26,8-136,8)$ & & $57,0(23,69-137,34)$ & \\
\hline Avaliação da saúde & & $<0,001$ & & 0,019 \\
\hline Excelente/Boa & 1,00 & & 1,00 & \\
\hline Razoável/Ruim & $3,83(2,14-6,84)$ & & $2,69(1,18-6,15)$ & \\
\hline \multicolumn{5}{|l|}{ Condição de saúde bucal } \\
\hline Necessidade de prótese & & 0,026 & & $0,374 * \star \star$ \\
\hline Não & 1,00 & & 1,00 & \\
\hline Sim & $2,48(1,11-5,54)$ & & $1,63(0,55-4,78)$ & \\
\hline
\end{tabular}

Nota: teste de Hosmer \& Lemeshow $(p=0,863)$.

* Análise univariada

** Ajustada para as características internas de cada bloco e pelo(s) bloco(s) superior(es);

*** Mantida para ajuste do modelo;

\# Salário mínimo em fevereiro de 2005 (R\$ 260,00).

bucal e maior capacidade de percepção tenham como reflexo maior relato e gravidade da interferência da condição bucal no cotidiano dos indivíduos 26. A percepção é fundamental na definição de qualidade de vida e está inserida no conceito proposto pela OMS 27 .

Identificar fatores associados a maior interferência da condição de saúde bucal no cotidiano dos indivíduos foi o eixo orientador de análise no presente estudo. A utilização do modelo hierárquico por intermédio da regressão logística múltipla permitiu identificar os fatores possivelmente determinantes para a interferência de maneira independente, seguindo os níveis hierárquicos teóricos de determinação. O teste de Hosmer \& Lemeshow indicou boa adequação do modelo final 24 .

A prevalência de algum tipo de interferência da condição bucal no cotidiano corrobora a literatura revisada, cuja freqüência varia entre $12,3 \% 28$ e 73,6\% 29. Diferenças nas faixas etárias e nas condições de saúde bucal podem contribuir para a variabilidade encontrada. A maioria dos estudos foi realizada em grupos de idosos, com maior percentual entre os edêntulos. A perda dental está associada a maior relato de 
interferência na maioria dos estudos 9,28,30,31. Questões culturais são também consideradas na análise ${ }^{28}$. Diferenças na valorização dos alimentos e nos padrões estéticos e de beleza, assim como as adversidades impostas pelo meio e a luta decorrente para satisfazer suas aspirações e necessidades são importantes fatores de explicação para as diferenças encontradas em estudos dessa natureza.

Para análise dos determinantes de maior interferência no cotidiano, as características sócio-demográficas, os aspectos relativos à percepção da saúde geral, saúde bucal, incluindo aqui o acesso aos serviços, e a condição clínica analisada segundo critérios clínicos foram considerados em blocos hierarquicamente sobrepostos. As características sócio-demográficas se encontram no nível distal de determinação e medeiam todo o desencadeamento do processo, condicionando a percepção e a saúde bucal dos indivíduos. Uma pior condição social relaciona-se com um menor acesso a medidas de controle, de prevenção e de tratamento das morbidades, resultando em piores condições de saúde. Paralelamente, os aspectos demográficos relacionam-se como características culturais e sociais, determinando maior capacidade de percepção dos agravos e das limitações impostas por eles 32 .

No presente estudo, a aglomeração domiciliar e o local de moradia estão associados à maior interferência, independente das demais características sócio-demográficas analisadas. As pessoas que residiam nas áreas definidas como as de pior condição de vida e que habitavam em domicílios com maior número de pessoas/cômodo referiram o dobro de interferência dos demais. Considera-se que esses indivíduos apresentavam pior condição bucal, com reflexos diretos em um pior desempenho das atividades diárias. Estudos nacionais e estrangeiros encontraram associação de superlotação no domicílio e local de residência com pior condição de saúde 5,33 .

A relação percepção e qualidade de vida indicada no próprio conceito da OMS 27 pode ainda contribuir na análise das variáveis auto-avaliação de saúde e dor orofacial. Ambas apresentaram associação significante com maior interferência da condição bucal no cotidiano independente da condição sócio-demográfica. A percepção de saúde é considerada consistente preditora de qualidade de vida 1 . Argumenta-se que debilidades na saúde geral e sintomas dolorosos na região orofacial aumentam a perspicácia dos indivíduos quanto às dificuldades do desempenho diário. Por outro lado, ambas podem contribuir para a ocorrência dessas dificuldades. A dor é co- mumente relatada entre indivíduos com saúde debilitada 34

A variável uso do fio dental mostrou-se associada à maior interferência, quando o bloco intermediário foi analisado separadamente dos demais, perdendo a significância estatística no modelo hierárquico, provavelmente devido às implicações sócio-econômicas desse tipo de hábito. Comportamento semelhante teve a variável acesso a serviços, que perdeu significância no bloco de percepção. Acesso aos serviços foi admitido como um aspecto relacionado com a percepção do indivíduo acerca de sua saúde; apresentou significância à análise bivariada, mas não permaneceu no modelo final. O fato de o principal sintoma dos agravos de saúde bucal ser a dor e igualmente ser a principal queixa da busca aos serviços odontológicos pode explicar a perda de significância no modelo, uma vez que estavam no mesmo nível hierárquico, prevalecendo a força da associação da dor sobre o acesso aos serviços de saúde.

Diferentemente das questões anteriores, a associação da variável sexo e interferências no desempenho diário apresenta controvérsias na literatura. As mulheres entrevistadas neste estudo relataram o dobro de alta interferência, corroborando os achados de McGrath et al. 29, McGrath \& Bedi 32 , Peek et al. 35 , Sanders \& Spencer 36. Outros estudos, no entanto, não encontraram associação estatisticamente significante ${ }^{37}$. Aspectos culturais e sociais têm permeado a discussão sobre essa temática, estando ambos associados à maior capacidade de percepção das mulheres, seja por padrões estéticos, seja pelas transformações biológicas comumente associadas a sintomatologias dolorosas, como a menarca. O papel de "cuidadoras", atribuído e assumido pelas mulheres no ambiente familiar e no contexto dos serviços de saúde, é outro argumento utilizado para explicar uma maior perspicácia de alterações no seu cotidiano.

No bloco proximal, nenhuma variável clínica, observada segundo critérios normativos, manteve-se independente dos blocos hierarquicamente superiores no modelo final. A influência do contexto social sobre aspectos comportamentais e psicológicos, e destes sobre o processo biológico, pode estar explicando esse comportamento 4. Piores condições sócio-econômicas, debilidades de saúde, hábitos e comportamentos deletérios têm se mostrado fortemente associados a precárias condições de saúde bucal 38,39,40. Vale ressaltar a já comentada associação entre dor e os agravos bucais. Sendo uma variável de percepção, a presença da variável dor alocada num nível hierarquicamente superior pode estar contribuindo para esse comportamento. 
Os resultados sugerem que os indicadores, baseados em critérios normativos, sejam insuficientes para mensurar a interferência da condição bucal no cotidiano dos indivíduos. Tal aspecto corrobora as reflexões da maioria dos autores que apontam debilidades do planejamento das ações de saúde orientado exclusivamente por tais indicadores 1,14,41. O sucesso do tratamento e a eficácia das medidas preventivas dependem diretamente da adesão dos indivíduos e da percepção dos mesmos sobre sua saúde e sobre os reflexos dela decorrentes. No estudo das necessidades de saúde bucal, portanto, é recomendável que a percepção dos indivíduos seja considerada simultaneamente à condição clínica 41 .

\section{Conclusões e considerações finais}

Interferência da condição bucal na qualidade de vida foi referida por um quinto da população do estudo, e o relato de alta interferência, por 11,4\%. A análise dos sintomas e das causas específicas

\section{Resumo}

O uso de indicadores subjetivos na odontologia vem se ampliando. Este estudo transversal de base populacional realizado em Chapecó, Santa Catarina, Brasil, analisou a relação entre condição bucal e qualidade de vida em adultos de 35 a 44 anos de idade $(n=622)$ e verificou a influência de: hábitos, visita ao dentista, auto-avaliação da saúde e características sócio-demográficas nesse processo. Usou-se uma amostra selecionada em múltiplos estágios e coleta com profissionais previamente treinados. A proporção de respostas foi de 81\%. Na análise dos fatores determinantes para baixa (Oral Impact on Daily Performance - OIDP $\leq 3$ ) e alta $(O I D P \geq 10)$ interferência da saúde bucal no cotidiano, utilizou-se modelo de regressão logística múltipla, não condicional, hierarquizada. A saúde bucal interferiu no cotidiano de $20,7 \%$ dos participantes e foi mais grave em 11,4\%. Morar em domicílios de maior aglomeração e áreas de pior condição de vida, ser mulher e sentir dor orofacial no último semestre compuseram fatores associados a maior interferência. É recomendável que a percepção dos indivíduos seja considerada simultaneamente à condição clínica na definição de ações dos serviços e de grupos prioritários ao atendimento em saúde bucal.

Saúde Bucal; Qualidade de Vida; Adulto; Desigualdades em Saúde da interferência remete à reflexão sobre o sofrimento envolvido no relato desse evento. Considera-se importante e merecedor de atenção esse tipo de informação no diagnóstico de saúde bucal da população.

O sofrimento e as limitações vivenciados são, possivelmente, características mais fiéis da condição de saúde bucal dos indivíduos que as seqüelas biológicas dos agravos e eventos mensurados pela maioria dos indicadores clínicos. Assim, os indicadores subjetivos são essenciais na definição das ações e dos grupos prioritários nos serviços de saúde para qualificar a informação da condição de saúde bucal oferecida pelos indicadores clínicos no planejamento dos serviços.

Os mecanismos desencadeadores dos reflexos da condição de saúde bucal na qualidade de vida merecem investigação continuada. Pesquisas qualitativas voltadas para o conhecimento dos aspectos envolvidos na percepção de saúde e sobre a capacidade dos indivíduos em lidar com as adversidades físicas e sociais certamente contribuirão para este aprimoramento.

\section{Colaboradores}

J. T. Lacerda participou na concepção, planejamento, análise e discussão dos resultados e aprovação da versão final. E. A. Castilho colaborou na concepção, planejamento, revisão crítica do conteúdo e aprovação da versão final. M. C. M. Calvo contribuiu na análise e discussão dos resultados, revisão crítica do conteúdo e aprovação da versão final. S. F. T. Freitas participou na análise e discussão dos resultados, revisão crítica do conteúdo e aprovação da versão final. 


\section{Referências}

1. Chen M, Hunter P. Oral health and quality of life in New Zealand: a social perspective. Soc Sci Med 1996; 43:1213-22.

2. Bjertness E. The important of oral hygiene on variation in dental caries in adults. Acta Odontol Scand 1991; 49:97-102.

3. Watt RG. Emerging theories into the social determinants of health: implications for oral health promotion. Community Dent Oral Epidemiol 2002; 30:241-7.

4. Holst D, Schüller AA. Oral health changes in an adult Norwegian population: a cohort analytical approach. Community Dent Oral Epidemiol 2000; 28:102-11.

5. Locker D. Deprivation and oral health: a review. Community Dent Oral Epidemiol 2000; 28:161-9.

6. Petersen PE. The World Oral Health Report 2003: continuous improvement of oral in the $21^{\text {st }}$ century - the approach of the WHO Global Oral Health Programme. Community Dent Oral Epidemiol 2003; 31 Suppl 1:3-23.

7. Paschoal SMP. Qualidade de vida do idoso: elaboração de um instrumento que privilegia sua opinião [Dissertação de Mestrado]. São Paulo: Faculdade de Medicina, Universidade de São Paulo; 2000.

8. Seidl EMF, Zannon CMLC. Qualidade de vida e saúde: aspectos conceituais e metodológicos. Cad Saúde Pública 2004; 20:580-8.

9. Sheiham A, Steele JG, Marcenes W, Tsakos G, Finch S, Walls AWG. Prevalence of impacts of dental and oral disorders and their effects on eating among older people; a national survey in Great Britain. Community Dent Oral Epidemiol 2001; 29:195203.

10. Locker D. Oral health and quality of life. Oral Health Prev Dent 2004; 2 Suppl 1:247-53.

11. Allison PJ, Locker D, Feine JS. Quality of life: a dynamic construct. Soc Sci Med 1997; 45:221-30.

12. Reisine ST. The impact of dental condition on social functioning and quality of life. Annu Rev Public Health 1988; 9:1-19.

13. McGrath C, Bedi R The association between dental anxiety and oral health-related quality of life in Britain. Community Dent Oral Epidemiol 2004; 32:67-72.

14. Leão A, Sheiham A. Relation between clinical dental status and subjectives impacts on daily living. J Dent Res 1995; 74:1408-13.

15. Reisine ST, Fertig J, Webwr J, Leder S. Impact of dental conditions on patients' quality of life. Community Dent Oral Epidemiol 1989; 17:7-10.

16. Foschiera MMP. Um pouco da história da construção do SUS em Chapecó. In: Franco TB, Peres MAA, Foschiera MMP, Panizzi M, organizadores. Acolher Chapecó: uma experiência de mudança do modelo assistencial, com base no processo de trabalho. São Paulo: Editora Hucitec; 2004. p. 55-69.

17. Cochran WG. Sampling techniques. 3rd Ed. New York: John Wiley and Sons; 1977.

18. Hair JF, Anderson RE, Tatham RL, Black WC. Análise multivariada de dados. 5a Ed. Porto Alegre: Editora Bookman; 2005.
19. Peres MA, Traebert J, Marcenes W. Calibração de examinadores para estudos epidemiológicos de cárie dentária. Cad Saúde Pública 2001; 17:153-9.

20. Landis JR, Koch GG. The measuremente of observer agreement for categorical data. Biometrics 1977; 33:159-74

21. Adulyanon S, Sheiham A. Oral impacts on daily performances. In: Slade GD, editor. Measuring oral health and quality of life. Chapel Hill: University of North Carolina; 1997.

22. Locker D, Grushka. The impact of dental and facial pain. J Dent Res 1987; 66:1414-7.

23. World Health Organization. Oral health survey: basic methods. Geneva: World Health Organization; 1997.

24. Hosmer DM, Lemeshow S. Applied logistic regression. New York: John Wiley; 1989.

25. Dahlgrem GE, Whitehead M. Policies and strategies to promote social equity in heath. Copenhagen: World Health Organization Regional Office for Europe; 1992.

26. Gift HC, Atchison KA, Drury TF. Perceptions of the natural dentition in the context of multiple variables. J Dent Res 1998; 77:1529-38.

27. The World Health Organization Quality of Life Assessment (WHOQOL): position paper from the World Health Organization. Soc Sci Med 1995; 41:1403-9.

28. Tsakos G, Marcenes W, Sheiham A. Cross-cultural differences in oral impacts on daily performance between Greek and British older adults. Community Dent Health 2001; 18:209-13.

29. McGrath CM, Bedi R, Gilthorpe MS. Oral health related quality of life-views of the public in the United Kingdom. Community Dent Health 2000; 17:3-7.

30. Adulyanon S, Vourapukjaru J, Sheiham A. Oral impacts affecting daily performance in a low dental disease Thai population. Community Dent Oral Epidemiol 1996; 24:385-9.

31. Steele JG, Sandres AE, Slade GD, Allen PF, Lathin S, Nuttall N, et al. How do age and tooth loss affect oral health impacts and quality of life? A study comparing two national samples. Community Dent Oral Epidemiol 2004; 32:107-14.

32. McGrath CM, Bedi R. Gender variations in the social impact $f$ oral health. J Ir Dent Assoc 2000; 46:87-91.

33. Antunes JLF, Narvai PC, Nugent ZJ. Measuring inequalities in the distribution of dental caries. Community Dent Oral Epidemiol 2004; 32:41-8.

34. Reyes-Gibby CC, Aday L, Cleeland C. Impact of pain on self-rated health in the community-dwelling older adults. Pain 2002; 95:75-82.

35. Peek CW, Gilbert GH, Duncan RP. Predictors of chewing difficulty onset among dentate adults: 24-month incidence. J Public Health Dent 2002; 62:214-21.

36. Sanders AE, Spencer AJ. Job characteristics and the subjective oral health of Australian workers. Aust N Z J Public Health 2004; 28:256-66. 
37. Gonçalves JR, Wassall T, Vieira S, Ramalho AS, Flório FM. Impacto da saúde bucal na qualidade de vida entre homens e mulheres. RGO (Porto Alegre) $2004 ; 52: 240-2$.

38. Lin HC, Schwarz E. Oral health and dental care in modern-day China. Community Dent Oral Epidemiol 2001; 29:319-28.

39. Hjern A, Grindefjord M, Sundberg H, Rosén M. Social inequality in oral health and use of dental care in Sweden. Community Dent Oral Epidemiol 2001; 29:167-74.
40. Henriksen BM, Axéll T, Laake K. Geographic differences in tooth loss and denture-wearing among the elderly Norway. Community Dent Oral Epidemiol 2003; 31:403-11.

41. Sheiham A, Cushing A, Maizels J. Developing sociodental indicators: the social impact of dental disease. Community Dent Health 1986; 3:3-17.

Recebido em 12/Mar/2007

Versão final reapresentada em 21/Dez/2007

Aprovado em 26/Dez/2007 